\title{
LA GESTION DES DÉCHETS RADIOACTIFS
}

\section{Éditorial}

\author{
Par M. Christian BATAILLE * \\ Député du Nord
}

uelle que soit l'option que l'on puisse avơr sur l'utilisation de l'énergie nucléaire, force est de constater que les déchets que cette industrie a engendrés existent et qu'il faudra bien un jour ou l'autre leur trouver une destination finale.

Tous les grands pays industrialisés ont d'ores et déjà mis en œuvre des programmes de recherches sur la gestion des déchets nucléaires à haute activité et sur leur stockage éventuel en couches géologiques profondes.

Même les pays qui, comme la Suède, ont en principe décidé de se passer à terme de l'énergie nucléaire ne peuvent échapper à la nécessité de trouver une destination finale à leurs déchets.

Prétendre, comme le font certains, qu'il est urgent d'attendre, constitue un aveu d'impuissance. Nous n'avons pas à laisser aux générations futures le soin de régler les problèmes que nous avons créés.

Dans le rapport de l'Office parlementaire d'évaluation que j'avais rendu après un an d'étude, d'auditions et de visites en France et à l'étranger, j'avais instamment demandé que toutes les solutions, enfouissement mais aussi retraitement poussé et transmutation, soient activement étudiées. Nous n'avons en effet pas le choix, sur un sujet aussi important, de faire l'impasse sur une solution si aléatoire qu'elle puisse être.

Mais j'avais également demandé que des garanties sérieuses soient apportées aux populations qui seront concernées par le stockage de ces déchets.
II faut être réaliste, il n'est jamais agréable d'avoir un dépôt de déchets dans son voisinage. Si en plus ces déchets sont radioactifs, les habitants concernés doivent être assurés que toutes les précautions possibles seront prises pour qu'à aucun moment leur santé ne soit mise en danger.

loi, votée en décembre 1991, très largement inspirée du rapport de l'Office, apporte sur ce point toutes les assurances nécessaires.

Les recherches devront porter en temps et avec autant d'insistance sur :

- la recherche de solutions permettant la séparation et la transmutation des éléments radioactifs à vie longue présents dans ces déchets;

- l'étude des possibilités de stockage réversible ou irréversible dans les formations géologiques profondes, notamment grâce à la réalisation de laboratoires souterrains ;

- l'étude de procédés de conditionnement et d'entreposage de longue durée en surface de ces déchets.

Mais, en outre, la loi précise qu'il ne s'agit pour le moment que de recherche et que les laboratoires souterrains ne pourront en aucun cas accueillir des déchets destinés au stockage.

La décision de créer, le cas échéant, un centre de stockage des déchets à haute activité ne pourra être prise que dans dix à quinze ans, par une nouvelle loi, et après la publication d'un rapport d'évaluation préparé par une commission d'experts indépendants.

-ors de la préparation du rapport et de la discussion du projet de loi, nous avons été, au Parlement, animés par une triple exigence de responsabilité, de transparence et de démocratie.

Responsabilité, car nous avons estimé que nous devons stocker nos déchets chez nous et dès maintenant sans renvoyer aux générations futures le soin de trouver une solution.

Transparence, car les années 1990 doivent marquer la fin de la culture du secre en matière nucléaire.

Démocratie, car il faut mettre fin aux méthodes administratives et autoritaires qui ont prévalu jusqu'ici. Aujourd'hui le public exige d'être informé et de participer aux décisions.

L'avenir de l'énergie nucléaire dépendra de notre capacité à résoudre le problème des déchets en respectant ces trois impératifs.

II ne faudrait pas, en effet, que "l'occassion" de la fin du cycle nucléaire, que certains cherchent à provoquer, finisse par remettre en question l'ensemble d'une industrie qui nous assure à la fois l'indépendance et la sécurité de notre approvisionnement en électricité.

- Auteur pour l'Office parlementaire d'évaluation des choix scientifiques et technologiques d'un rapport sur la gestion des déchets nucléaires à haute activité. Rapporteur du projet de loi relatif aux recherches sur la gestion des déchets radioactifs. 


\section{$\begin{array}{llllllllllllll}U & N & S & A & V & O & I & R & - & F & A & I & R & E\end{array}$ $\begin{array}{llllllllllll}E & X & C & E & P & T & I & O & N & N & E & L\end{array}$}

Pendant trente ans, l'industrie nucléaire française a optimisé la gestion des déchets radioactifs, s'adaptant à l'évolution des réglementations, répondant aux besoins et respectant les préoccupations du public en matière d'environnement. Soutenue par ses sociétés mères, CEA et Cogema, et grâce aux accords passés avec l'EDF et l'Andra (Agence nationale pour la gestion des déchets radioactifs), SGN a pu étendre son savoir-faire sans pareil et profiter du retour d'expérience de l'ensemble de l'industrie nucléaire française dans le domaine du traitement, conditionnement et stockage définitif des déchets.
Des systèmes fiables et économiques pour une gestion sûre des déchets radioactifs

\section{SGN : l'expérience française en matière de gestion de déchets radioactifs.}

\section{Services de conseil}

Ces services permettent de définir l'ensemble du problème jusqu'au stockage définitif, de proposer des méthodes de caractérisation des déchets, des critères d'acceptation des colis conditionnés, des systèmes d'identification et de suivi. Ils permettent également de préparer des rapports de sécurité et des programmes d'information du public. Récemment, de nombreux clients ont réclamé ce type de service, notamment Tepco et un groupe de sociétés japonaises, l'AEC de Taiwan, l'Etat de l'Illinois et l'ORNL aux Etats-Unis.

\section{Traitement et conditionnement des} déchets

SGN a développé des méthodes de traitement et de conditionnement pour tous les types de déchets et met sa très longue expérience d'ingénierie au profit de l'étude et de la réalisation du système industriel le mieux adapté aux types, volumes produits et conditions de coûts. Ces services ont été fournis à Eurochemic en Belgique, à KAERI et Kopec en Corée, à SKB et Sydkraft en Suède, à PNC et JAERI au Japon, à Batan en Indonésie, à BNFL au Royaume Uni, à Enresa en Espagne, à ENEA en Italie, à TPC à Taiwan et enfin, à BWFC, Duke Power Co. et Rockwell aux Etats-Unis, pour ne citer que quelques exemples. SGN fournit aussi des systèmes standard comme les unités fixes ou mobiles pour le bitumage, le bétonnage ou le compactage. Ces unités sont utilisées par Japco et Kepco au Japon et par Virginia Power Co. aux Etats-Unis.

\section{Systèmes de mesure}

SGN propose également une gamme complète de systèmes de mesure faisant appel à l'analyse passive et aux méthodes actives pour évaluer l'activité et la composition en radioélé- ments des déchets. Ces systèmes, comme ceux fournis au CEA à Fontenay-aux-Roses et à Cadarache, permettent une analyse précise, en particulier des très faibles teneurs en émetteurs alpha dans les colis de déchets.

\section{Stockage des déchets}

Par ailleurs, SGN assure la diffusion du savoir-faire français, exemplaire, en matière de stockage définitif des déchets de faible activité (par exemple, pour le compte du CNSI aux Etats-Unis) et de stockage intérimaire des déchets de haute activité (par exemple, pour le compte de la SEP aux Pays-Bas).

Du conseil à l'assistance à l'exploitation en passant par l'ingénierie et la gestion de projets, les services fournis par SGN contribuent à répandre dans le monde entier le savoir-faire et l'expérience française en matière de gestion des déchets radioactifs.

\section{SCV}

RESEAU EURISYS

SGN, Société générale pour les techniques nouvelles

1 , rue des Hérons - Montigny-le-Bretonneux, 